\title{
Channel Estimation of IRS-Aided Communication Systems with Hybrid Multiobjective optimization
}

\author{
Zhen Chen*, Jie Tang*, Hengbin Tang*, Xiuyin Zhang*, Daniel Ka Chun So ${ }^{\dagger}$ and Kai-Kit Wong ${ }^{\ddagger}$ \\ * School of Electronic and Information Engineering, South China University of Technology, Guangzhou, China, \\ ${ }^{\dagger}$ School of Electrical and Electronic Engineering, University of Manchester, Manchester, United Kingdom, UK \\ ${ }^{\ddagger}$ Department of Electronic and Electrical Engineering, University College London, UK
}

\begin{abstract}
In this paper, we propose a compressive channel estimation techniques for IRS-assisted mmWave multi-input and multi-output (MIMO) system. To reduce the training overhead, the inherent sparsity in mmWave channels is exploited. By utilizing the properties of Kronecker products, IRS-assisted mmWave channel estimation are converted into a sparse signal recovery problem, which involves two competing cost function terms (measurement error and a sparsity term). Existing sparse recovery algorithms solve the combined contradictory objectives function using a regularization parameter, which leads to a suboptimal solution. To address this concern, a hybrid multiobjective evolutionary paradigm is developed to solve the sparse recovery problem, which can overcome the difficulty in the choice of regularization parameter value. Simulation results show that under a wide range of simulation settings, the proposed algorithm achieves competitive error performance compared to existing channel estimation algorithms.
\end{abstract}

\section{INTRODUCTION}

$\mathbf{I}$ NTELLIGENT reflecting surface (IRS) is an emerging technology to improve the spectrum efficiency of wireless networkswith minimal energy consumption [1]-[3]. By jointly adjusting the reflected signal amplitude and/or phase shift at each of the IRS elements according to the dynamic wireless channels, the signals reflected by IRS and propagated through other paths can be constructively combined at the intended receiver to enhance the received signal power. In addition, since it is not equipped with any signal processing equipments such as analog-to-digital, digital-to-analog converter and modulator/demodulator, IRS is with low complexity and can be easily deployed on urban commercial buildings, ceilings or indoor spaces [3]. These significant advantages make IRS passive beamforming as a green energy-efficient technique for beyond 5G (B5G) cellular Internet of Things (IoT), and hence it has been investigated in different communication scenarios such as multi-cell cellular networks, secure massive MIMO transmission, simultaneous wireless information and power transfer (SWIPT) [4], [5] etc.

However, the implementation of IRS-Assisted millimeterwave (mmWave) MIMO communications is quite challenging in practice. One of the key challenge is the efficient channel estimation to acquire the CSI. A vast majority of the existing works assume the availability of perfect CSI to design the precoding vectors at the base station (BS) and phase shifts matrix at the IRS. Nevertheless, this assumption is difficult to realize in practice. In particular, as opposed to conventional backscatter and relay-assisted communication systems, where channels can be estimated using transmission/processing pilot symbols, the IRS can only reflect signals without the capabilities of signal transmission/processing, and hence it is practically difficult to estimate their channels.

It is worth mentioning that since millimeter wave channels tend to have only a few significant paths and directional beamforming, a large number of antennas is essentially required to mitigate the severe attenuation [6]. Therefore, the compressed sensing (CS) techniques is applied for the mmWave channels estimation, which can be leveraged to effectively estimate mmWave channels in angular domain of massive MIMO channels [7]-[9]. By exploiting the sparsity of mmWave channels, some CS-based mathods have been proposed for channel estimation, which can significantly reduce the required pilot overhead [10], such as the least absolute shrinkage and selection operator (LASSO) algorithm [11], [12]. It is particularly beneficial to MIMO systems, in which the number of pilot symbols increases linearly with the number of transmit antennas.

However, existing CS-based model are effective only when the number of transmit antennas is sufficiently large to guarantee the desired sparsity level. Such antenna configuration is unrealistic when there are massive amount of low-rate devices in the network. In addition, the performance of CS-based recovery method is usually dependent on the regularization parameter. Over the recent few years, various strategies for choosing regularization parameter have been studied [13], [14]. One possible method of avoiding the difficulties is to convert the constrained optimization problem into multiobjective optimization problems (MOPs), and the corresponding multiobjective evolutionary algorithms (MOEAs) is employed to yield better results than the use of conventional single objective optimization methods. It is worth noting that the aforementioned works cannot be extended directly to IRSbased mmWave channel estimation, due to the fact that the mmWave channel with IRS will make the problem highly complex and difficult to solve, which is one of the major motivations to devote our endeavour to developing efficient channel estimation strategies for IRS-assisted mmWave communication MIMO systems.

In this work, we propose a sparse channel estimation scheme that combines CS techniques and a hybrid multiobjective evolutionary optimization method for IRS-assisted mmWave 
massive MIMO systems. The key observation for the proposed scheme are exploiting CS-based sparse channel estimation to reduce the training overhead and to find an optimal tradeoff between competing objective functions (sparsity and measurement error) in CS-based sparse recovery model. According to the MOPs, the final solution of the proposed channel estimation is selected in the knee areas, which indicates the best balance between the measurement error and the sparsity constraint whilst providing the best performance in sparse channel estimation.

The rest of this paper is organized as follows. In Section II, the detailed description of CS-based channel estimation model is proposed. Section III proposes a hybrid multiobjective evolutionary approach for mmWave channel estimation. The simulation results on the performance of the proposed method are reported in Section IV. Section V concludes the paper.

Notations: The boldface lower-case and upper-case symbols denote vectors and matrices respectively. $\operatorname{vec}(\mathbf{A})$ is a vector obtained through the vectorization of a matrix $\mathbf{A}$, and $\operatorname{vec}^{-1}(\mathbf{A})$ represents a matrix obtained by the inverse of vectorization. $\|\boldsymbol{a}\|_{p}$ represents the $p$-norm of $\boldsymbol{a}$, where $a_{i}$ is the $i$-th component of $\boldsymbol{a}$ and $p$ denotes an integer. The superscripts $(\cdot)^{T},(\cdot)^{H}$ and $(\cdot)^{*}$ represent the transpose, conjugate transpose and complex conjugat respectively. The Kronecker product of two matrices $\boldsymbol{X}$ and $\boldsymbol{Y}$ is denoted as $\boldsymbol{X} \otimes \boldsymbol{Y} . \mathbb{R}$ and $\mathbb{C}$ denote the set of real and complex numbers, respectively.

\section{System Model ANd PRoblem Formulation}

In this section, the IRS-assisted mmWave MIMO communication system is introduced. Then, we formulate the mmWave channel estimation problem as a CS-based sparse recovery problem in the following.

\section{A. System Model}

We focus on the downlink transmission of an IRS-assisted mmWave MIMO system in Fig. 1, where an IRS is deployed to assist the data transmission from the BS to a user equipment (UE). Without loss of generality, it is assumed that the IRS is a planar array with $M$ reflecting elements and the signals are reflected by the IRS once. The IRS is attached to the facade of a building located in the LoS link of the BS. The phase shifts of each reflecting element on the IRS are configurable via a smart controller, which gets this information from the BS over a backhaul link. The BS is equipped with $N_{B S}$ antennas. Let $\boldsymbol{R} \in \mathbb{C}^{N_{I R S} \times N_{B S}}$ be the channel from the BS to the IRS, $\boldsymbol{H}_{r} \in \mathbb{C}^{N_{U E} \times N_{I R S}}$ denotes the channel from the IRS to the UE. This is a valid assumption as IRS is practically used to mainly support UE without direct communication link from the BS due to deep path loss or obstacle blockage. Furthermore, a time-division duplexing (TDD) scheme is used for the downlink CSI acquisition by utilizing the characteristic of the channel reciprocity.

For the channel training scheme in the above mentioned system, we suppose that each time frame contains $T$ time slots and the BS uses $P$ different precoding vectors at $P$ successive time frame. At the receiver side, the UE employs a combining

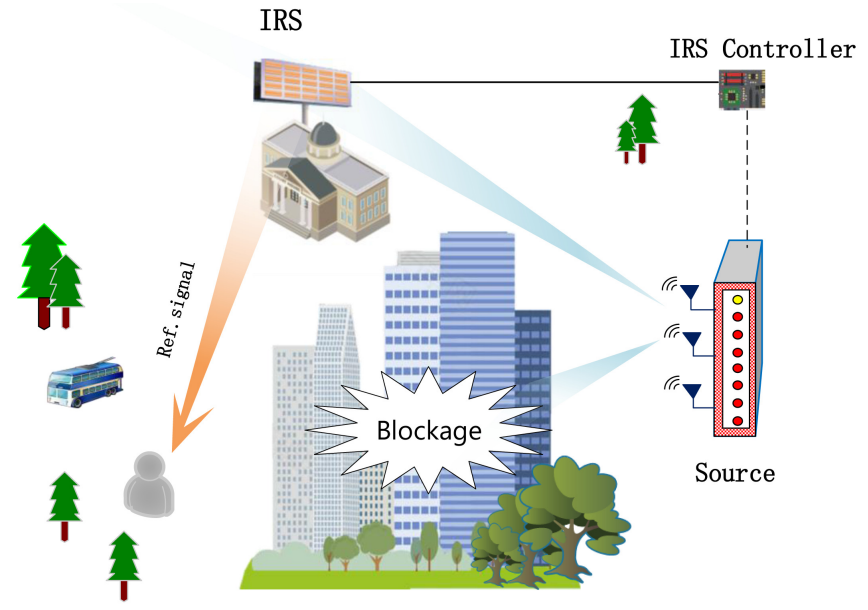

Fig. 1: Illustration of the considered IRS-assisted millimeter-wave MIMO communication systems.

vector $\boldsymbol{w}(t) \in \mathbb{C}^{N_{U E}}$ to combine the received signal at time slot $t$, then the signal received by the UE can be expressed as

$$
\boldsymbol{y}_{p}(t)=\boldsymbol{w}^{H}(t) \boldsymbol{H} \boldsymbol{x}_{p}(t)+\boldsymbol{n}(t),
$$

where $\boldsymbol{n}(t) \in \mathbb{C}^{N_{U E}}$ is the additive white Gaussian noise with zero mean and variance $\sigma^{2}$, and $\boldsymbol{H}$ is the cascaded channel matrix of the IRS that is given by

$$
\boldsymbol{H}=\boldsymbol{H}_{r} \boldsymbol{\Theta} \boldsymbol{R},
$$

where $\Theta$ is diagonal phase-shifting matrix of the IRS, which can be expressed as

$$
\boldsymbol{\Theta}=\operatorname{diag}\left(\beta_{1} e^{j \theta_{1}}, \ldots, \beta_{M} e^{j \theta_{M}}\right),
$$

and $\theta_{m} \in[0,2 \pi), \beta_{m} \in[0,1]$ being the phase shift and amplitude reflection coefficient associated with $m$-th reflecting element of the IRS, respectively. To maximize the reflection power of the IRS and simplify its hardware design, we consider the same $\beta_{m}, \forall m$ for all the reflecting elements of the IRS.

With regard to the total $T$ time frames, the identical combiner $\boldsymbol{W} \in \mathbb{C}^{N_{U E} \times Q}$ is utilized by stacking $\boldsymbol{w}_{t}$ with $t=1, \ldots, T$, and thus the received matrix at the UE side can be written as

$$
\boldsymbol{Y}=\boldsymbol{W}^{H} \boldsymbol{H} \boldsymbol{X}+\boldsymbol{N},
$$

where $\boldsymbol{W}=\left[\boldsymbol{w}_{1}, \boldsymbol{w}_{2}, \ldots, \boldsymbol{w}_{T}\right]$ is a $N_{U E} \times Q$ combiner matrix, $\boldsymbol{Y}=\left[\boldsymbol{y}_{1}, \boldsymbol{y}_{2}, \ldots, \boldsymbol{y}_{T}\right]$ is a $Q \times T$ received matrix, $\boldsymbol{X}=$ $\left[\mathbf{x}_{1}, \mathbf{x}_{2}, \ldots, \mathbf{x}_{T}\right]$ is a $N_{B S} \times T$ beamforming matrix, $\boldsymbol{N}=$ $\left[\boldsymbol{n}_{1}, \boldsymbol{n}_{2}, \ldots, \boldsymbol{n}_{T}\right]$ is a $Q \times T$ noise matrix by concatenating the noise vectors, respectively.

\section{B. Problem Formulation}

Before formulating the channel estimation problem, the channel model for the IRS-assisted mmWave MIMO communication system should be described first. By smartly adjusting the phase shifts of all scattering elements, as illustrated in Fig. 1 , the superposed signal arrived at the IRS will be directly reflected towards the desired UEs without any additional 
processing, and thus there is no signal processing delay. According to the discussion above, we take into account the cascade channel matrix $\boldsymbol{H}$, which is divided into the BS-IRS channel $\boldsymbol{R}$ and the IRS-UE channel $\boldsymbol{p}$. The main challenge is to estimate the BS-IRS channel and the IRS-UE channel simultaneously.

To be specific, the BS-IRS channel can be modeled as [15]

$$
\boldsymbol{R}=\sqrt{\frac{N_{B S} N_{I R S}}{\rho}} \sum_{l=1}^{L} \alpha_{l} \boldsymbol{a}_{I R S, 1}\left(\varphi_{l}, \gamma_{l}\right) \boldsymbol{a}_{B S}^{H}\left(\phi_{l}\right),
$$

where $\alpha_{l}$ is the channel complex gain of the $l$-th path, $L$ denotes the scattering paths of BS-IRS link, $\rho$ denotes the path loss between the BS and IRS, $\varphi_{l}\left(\gamma_{l}\right)$ denotes the azimuth (elevation) angle of arrival (AoA), $\phi_{l}$ is the angle of departure (AoD) of the $l$-th path; $\boldsymbol{a}_{I R S, 1}$ and $\boldsymbol{a}_{B S}$ denote the array response vectors associated with the IRS and BS, respectively.

Due to the sparse scattering nature of mmWave channels, the number of path $L$ is small relative to the dimensions of $\boldsymbol{R}$. The corresponding $\boldsymbol{R}$ can be expressed as

$$
\boldsymbol{R}=\left(\boldsymbol{F}_{x} \otimes \boldsymbol{F}_{y}\right) \boldsymbol{\Sigma}_{\alpha} \boldsymbol{F}_{B S}^{H} \triangleq \boldsymbol{F}_{I R S, 1} \boldsymbol{\Sigma}_{\alpha} \boldsymbol{F}_{B S}^{H},
$$

where $\boldsymbol{F}_{x} \in \mathbb{C}^{M_{x} \times M_{L, x}}$ and $\boldsymbol{F}_{y} \in \mathbb{C}^{M_{y} \times M_{L, y}}$ are similarly defined with each of its columns having a form of $\boldsymbol{a}_{x}(u)$ and $\boldsymbol{a}_{y}(v)$, respectively. $\boldsymbol{\Sigma}_{\alpha} \in \mathbb{C}^{M_{L} \times N_{L}}$ is a sparse matrix with $L$ non-zero entries corresponding to the channel path gains $\left\{\sqrt{\frac{N_{B S} N_{I R S}}{\rho}} \alpha_{l}\right\}, \boldsymbol{F}_{B S} \in \mathbb{C}^{N_{B S} \times N_{L}}$ is an overcomplete matrix $\left(N_{L} \geq N_{B S}\right)$ and each of its columns has a form of $\boldsymbol{a}_{B S}\left(\phi_{l}\right)$, with $\phi_{l}$ chosen from a pre-discretized grid.

Similar to the BS-IRS channel $\boldsymbol{R}$, the IRS-UE channel can be modeled as

$$
\boldsymbol{H}_{r}=\boldsymbol{F}_{U E} \boldsymbol{\Sigma}_{c}\left(\boldsymbol{F}_{x} \otimes \boldsymbol{F}_{y}\right)^{H} \triangleq \boldsymbol{F}_{U E} \boldsymbol{\Sigma}_{c} \boldsymbol{F}_{I R S, 2}^{H} .
$$

With the property of Khatri-Rao product, substituting (6) and (7) into (2), the cascade channel model can be expressed as

$$
\begin{gathered}
\operatorname{vec}(\boldsymbol{H})=\operatorname{vec}\left(\boldsymbol{F}_{U E} \boldsymbol{\Sigma}_{c} \boldsymbol{F}_{I R S, 2}^{H} \boldsymbol{\Theta} \boldsymbol{F}_{I R S, 1} \boldsymbol{\Sigma}_{\alpha} \boldsymbol{F}_{B S}^{H}\right) \\
\stackrel{(a)}{=}\left(\boldsymbol{F}_{B S}^{*} \otimes \boldsymbol{F}_{U E}\right)\left(\boldsymbol{\Sigma}_{\alpha}^{T} \otimes \boldsymbol{\Sigma}_{c}\right) \boldsymbol{D} \boldsymbol{u}
\end{gathered}
$$

where (a) comes from $\boldsymbol{u} \triangleq\left[e^{j \theta_{1}}, \ldots, e^{j \theta_{M}}\right]^{H} \in \mathbb{C}^{M}$ and $\boldsymbol{D}=$ $\boldsymbol{F}_{I R S, 1}^{T} \otimes \boldsymbol{F}_{I R S, 2}^{H}$.

Thus, the received signal $\boldsymbol{y}=\operatorname{vec}(\boldsymbol{Y})$ in (4) can be written as

$$
\begin{aligned}
\boldsymbol{y} & =\left(\boldsymbol{X}^{T} \otimes \boldsymbol{W}^{H}\right) \operatorname{vec}(\boldsymbol{H})+\operatorname{vec}(\boldsymbol{N}) \\
& \stackrel{(a)}{=}(\boldsymbol{D} \boldsymbol{u})^{T} \otimes\left(\left(\boldsymbol{X}^{T} \otimes \boldsymbol{W}^{H}\right) \otimes\left(\boldsymbol{F}_{B S}^{*} \otimes \boldsymbol{F}_{U E}\right)\right) \boldsymbol{h}+\operatorname{vec}(\boldsymbol{N}),
\end{aligned}
$$

where (a) is obtained by $\boldsymbol{h} \triangleq \operatorname{vec}\left(\boldsymbol{\Sigma}_{\alpha}^{T} \otimes \boldsymbol{\Sigma}_{c}\right)$ and the property of Khatri-Rao product.

Based on this result, the (9) can be further expressed as

$$
\boldsymbol{y}=\boldsymbol{\Phi} \boldsymbol{h}+\boldsymbol{n},
$$

where $\boldsymbol{\Phi} \triangleq\left(\boldsymbol{D}_{L} \boldsymbol{u}\right)^{T} \otimes\left(\left(\boldsymbol{X}^{T} \otimes \boldsymbol{W}^{H}\right) \otimes\left(\boldsymbol{F}_{B S}^{*} \otimes \boldsymbol{F}_{U E}\right)\right)$ and $\boldsymbol{n}=\operatorname{vec}(\boldsymbol{N})$.

Based on the structure properties of (10), the channel estimation is a classic CS-based sparse recovery problem [16], and thus the CS theory can be employed to estimate $\boldsymbol{h}$ by solving the following sparse recovery problem:

$$
\min _{\boldsymbol{h}}\left\{\boldsymbol{G}(\boldsymbol{h})=\frac{1}{2}\|\boldsymbol{y}-\boldsymbol{\Phi} \boldsymbol{h}\|_{2}^{2}+\lambda\|\boldsymbol{h}\|_{p}\right\},
$$

where $\lambda$ is regularization parameter and $p$ is usually set to 0 or 1 .

It is found that two optimization objectives of the optimization problem (11) are conflicting to each other. Especially, it is difficult to choose the optimal $\lambda$, since different regularization parameter $\lambda$ in the problem (11) will yield different optimal solutions. It is interesting that this deficiencies can be naturally alleviated by employing MOEAs. Next, we will present a hybrid MOEA and show how it can be used to solve the IRSassisted channel estimation problem.

\section{Multiobjective Evolutionary Approach to CHANNEL ESTIMATION}

In this section, considering the measurement error and sparse constraint as two conflicting objectives, we transform the single objective problem (11) to a bi-objective optimization problem, and a hybrid evolutionary algorithm is proposed by incorporating a IST-based local search to achieve multiobjective optimization model. Therefore, the CS-based sparse recovery problem (11) can be formulated as a bi-objective optimization problem

$$
\min _{\boldsymbol{h}} \boldsymbol{G}(\boldsymbol{h})=\min _{\boldsymbol{h}}\left(f_{1}(\boldsymbol{h}), f_{2}(\boldsymbol{h})\right),
$$

where $f_{1}(\boldsymbol{h})=\frac{1}{2}\|\boldsymbol{y}-\boldsymbol{\Phi} \boldsymbol{h}\|_{2}^{2}$ denotes measurement error term and $f_{2}(\boldsymbol{h})=\|\boldsymbol{h}\|_{0}$ denotes sparse constraint term.

A hybrid MOEA based on differential evolution (DE) is presented to solve the above problem (12). The procedure of the proposed algorithm is given in Algorithm 1. Let $P^{t}=$ $\left[\boldsymbol{h}_{1, t}, \boldsymbol{h}_{2, t}, \ldots, \boldsymbol{h}_{N, t}\right]$ be the current population with size of $N$ solutions, where $\boldsymbol{h}_{i, t}$ denotes the $i$-th solution of the population and $t$ denotes the generation to which the population belongs. $P_{\text {mut }}^{t}$ and $P_{\text {Cro }}^{t}$ are the number of individuals for mutation and crossover operators, respectively, which are obtained by the corresponding mutation and crossover rates. The mutation and crossover operators are applied to generate new individuals. Then, an iterative soft-thresholding (IST)-based local search operator is used to improve the performance of the algorithm. If the termination condition is satisfied, the optimal Pareto front $(\mathrm{PF})$ of algorithm is obtained. For decision process, the angle-based method is used to determine the knee points of the problem. The detailed description about the complete algorithm is summarized as follows.

\section{A. Mutation and crossover operators}

Mutation and crossover mean to generate the new individuals and the corresponding offspring solution controlled by mutation factor and crossover parameter. The mutation factor $F$ and crossover factor $C$ largely affect the searching ability and premature convergence of algorithm. Therefore, adaptive DE algorithm or its variations are exploited, e.g., self-adaptive DE (SaDE) [17]. In DE, the random value is 


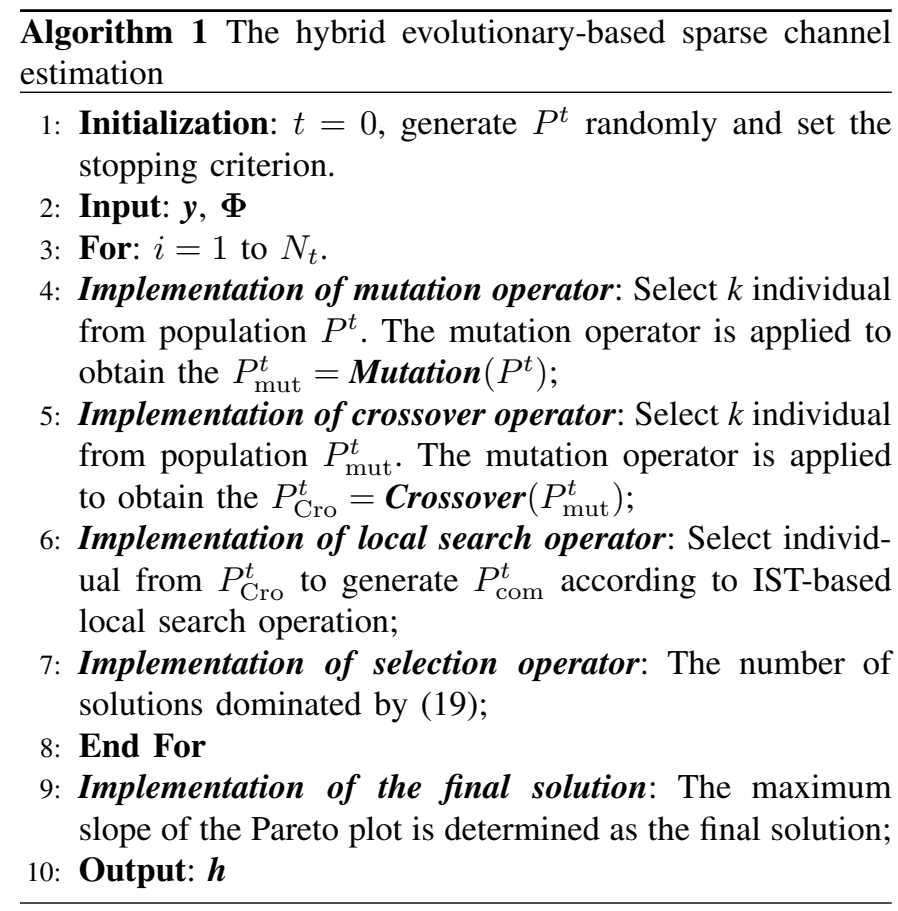

usually used in crossover operator to dynamically generate its offspring. However, this random nature may lead to instability of offspring solution. To address the concern, an improved mutation and crossover operation is exploited to generate promising solutions. We adopt a parameter-adaptive mechanism for mutation operation, which is given as follows

$$
\boldsymbol{v}_{i, t}=\boldsymbol{h}_{i, t}+F\left(\boldsymbol{h}_{r 2, t}-\boldsymbol{h}_{r 3, t}\right),
$$

where the $\boldsymbol{h}_{r 2, t}$, and $\boldsymbol{h}_{r 3, t}$ are randomly selected within the current population $P^{t}$ and they are also different from $\boldsymbol{h}_{i}^{t}$. The corresponding mutation factor $F$ is used to scale the difference vectors, that is

$$
F= \begin{cases}F_{0}, & \text { if iteration }=1 \\ F_{0} e^{-2 t / G_{\max }} & \text { otherwise }\end{cases}
$$

and $F_{0}$ is the initial value and $G_{\max }$ is the maximum iterations.

After the mutation operation, a crossover operator is carried out on $\boldsymbol{v}_{i, t}$ and $\boldsymbol{h}_{i, t}$ to generate a trial vector, which is expressed as follows

$$
\boldsymbol{z}_{i, t}= \begin{cases}\boldsymbol{v}_{i, t}, & \text { if } r \leq C \\ \boldsymbol{h}_{i, t} & \text { otherwise }\end{cases}
$$

where $r$ is a random number within the interval $[0,1]$.

\section{B. IST-based local search operator}

In order to generate better new individuals, we develop an additional step beyond crossover and mutation, where the generated individuals are further perturbed using a local search strategy improved from IST [11]. To be specific, we introduce the IST algorithm in detail and then describe how the local search strategy is incorporated within the unconstrained optimization problem (11), which can be treat as a special case of the following problem:

$$
\min _{z_{i, t}}\left\{\boldsymbol{g}\left(\boldsymbol{z}_{i, t}\right)=f_{1}\left(\boldsymbol{z}_{i, t}\right)+\lambda f_{2}\left(\boldsymbol{z}_{i, t}\right)\right\}, \quad \boldsymbol{z}_{i, t} \in P_{\text {Cro }}^{t}
$$

where $f_{1}\left(\boldsymbol{z}_{i, t}\right)$ is a smooth and convex function, $f_{2}\left(\boldsymbol{z}_{i, t}\right)$ is a separable function but not necessarily smooth nor convex, and $\lambda$ is a Lagrangian multiplier that balances the tradeoff between the measurement error and the sparse constraint term.

The optimization problem (16) is solved through a sequence of iterations $\left\{z_{i, t}^{(k)}, k=1,2, \ldots, K\right\}$, where $z_{i, t}^{(k+1)}$ is obtained from the previous solution $z_{i, t}^{(k)}$ by optimizing the following optimization subproblem:

$$
\boldsymbol{z}_{i, t}=\min _{\boldsymbol{z}_{i, t}} \frac{1}{2}\left\|\boldsymbol{z}_{i, t}-\boldsymbol{d}_{i, t}^{(k)}\right\|_{2}^{2}+\frac{\lambda}{\rho_{i}^{(k)}} f_{2}\left(\boldsymbol{z}_{i, t}\right),
$$

where the diagonal matrix $\rho_{i}^{(k)} \boldsymbol{I}$ is an estimation of the Hessian matrix $\nabla^{2} f_{1}\left(z_{i, t}^{(k)}\right)$ and

$$
\hat{\boldsymbol{d}}_{i, t}^{(k)}=\boldsymbol{z}_{i, t}^{(k)}-\frac{1}{\rho_{i}^{(k)}} \nabla f_{1}\left(\boldsymbol{z}_{i, t}^{(k)}\right) .
$$

Accordingly, the iterative soft/hard thresholding [18] can be used to solve the problem (18).

\section{Selection operator}

The selection scheme also differs from that of existing DE algorithms. The solution of current population is selected to generate the next population, and its corresponding trial solution is given by using the following rule:

$$
\boldsymbol{h}_{i, t+1}=\left\{\begin{array}{cc}
\boldsymbol{s}_{i, t} & \text { if } \quad \boldsymbol{g}\left(\boldsymbol{s}_{i, t}\right) \leq \boldsymbol{g}\left(\boldsymbol{z}_{i, t}\right), p=0 \\
\boldsymbol{z}_{i, t} & \text { otherwise }
\end{array}\right.
$$

where $\boldsymbol{s}_{i}^{t}$ is the output result of local search operation.

By using the (19), each solution of the temporary (trial) population is compared with its counterpart in the current population. The tournament selection is carried out to select the lower objective function value that will survive to the population of the next generation. As a result, it is guaranteed that all the solutions of the next generation are as well as or better than the current generation. It is worth noting that trial solution is not compared against all the solutions in the current population, but only against its counterpart.

\section{Acquisition of the final solution}

In MOPs, the Pareto knee region provides promising tradeoff between the two conflicting objectives, and the near optimal solution is chosen in this region. It proves that the Pareto knee point can be regarded as a reasonable final solution [19]. Next, we choose the solution corresponding to the maximal slope change as the Pareto knee point. The basic idea is to determine the final solution by the maximum slope of the Pareto plot at a given point. Thereafter, the solution with the maximum of slope variance $\Delta \phi$ value is considered as the nearly optimal solution.

\section{NUMERICAL RESULTS}

In this section, we evaluate the performance of the proposed channel estimation scheme for mmWave MIMO systems with IRS. The performance of proposed hybrid evolutionary-based 


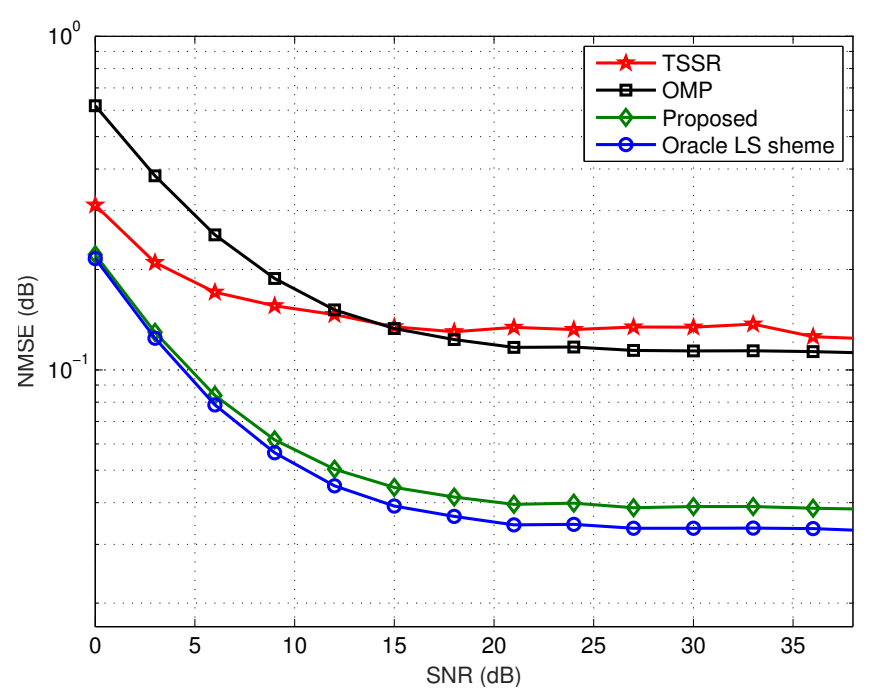

Fig. 2: NMSEs of respective algorithms versus the SNR $\left(N_{t}=196\right)$.

MOEA is experimentally compared to the existing channel estimation algorithms, i.e., orthogonal matching pursuit (OMP) [9], two-stage estimation exploiting both sparsity and low rankness (TSSR) [20]. The oracle LS scheme is considered as our benchmark. For the benchmark mmWave systems, we assume that the IRS is equipped with $M=32$ reflecting elements. The number of antennas at the BS is set to be $N_{t}=256$ to serve the UE. The carrier frequency is $f_{c}=28$ GHz. The distance between neighboring antenna elements is set as the half-wavelength of the signal.

To evaluate the performance of channel estimation schemes, we compare the considered CSI estimation techniques in terms of both the normalized mean square error (NMSE). The NMSE is considered to quantify the accuracy of channel estimation, which is mathematically defined as

$$
\mathrm{NMSE} \triangleq 10 \log _{10} \frac{\|\boldsymbol{h}-\hat{\boldsymbol{h}}\|_{2}^{2}}{\|\boldsymbol{h}\|_{2}^{2}}
$$

where $\hat{\boldsymbol{h}}$ denotes the estimation for the true channel $\boldsymbol{h}$ with the considered techniques. Apparently, when the value of NMSE is smaller, the estimated channel $\hat{\boldsymbol{h}}$ is closer to the true channel $\boldsymbol{h}$. In other words, the result of NMSE is zero, which indicates that $\hat{\boldsymbol{h}}$ is the perfect estimation of $\boldsymbol{h}$.

In the first simulation, the performance gain of our proposed strategy for channel estimation is studied, and some benchmark schemes are introduced in the simulation based performance comparison and analysis. In Fig. 2 and Fig. 3, we compare the NMSEs of different channel estimation schemes versus the SNR levels. It can be observed that the oracle LS estimator provides the best achievable performance for all channel estimation method. On the other hand, one can note that, using the hybrid MOEA for IRS-based system, the proposed algorithm can obtain significant NMSE improvement compared with conventional OMP and TSSR algorithms. In addition, one can observe that the proposed algorithm performs remarkably with a small gap compared to the benchmark. These results demonstrate that the IRS is a promising tech-

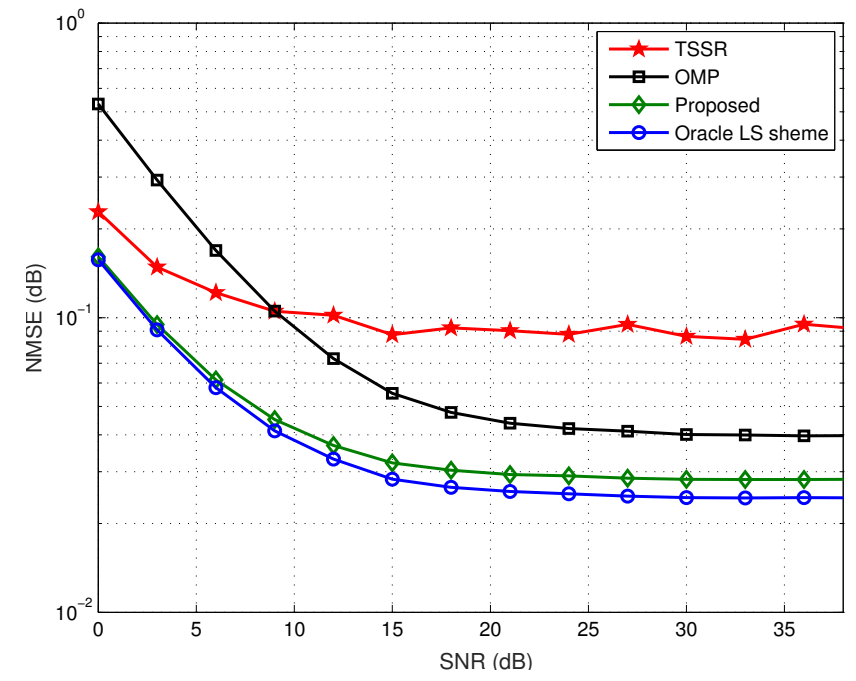

Fig. 3: NMSEs of respective algorithms versus the $\operatorname{SNR}\left(N_{t}=256\right)$.

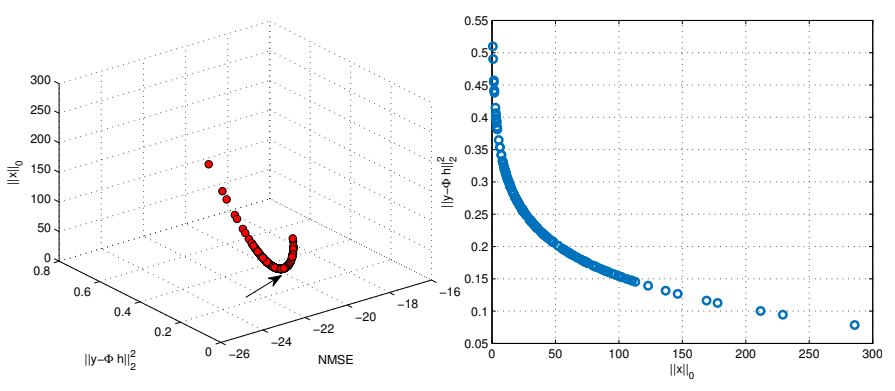

Fig. 4: Relationship between NMSE, measurement error and the sparsity of the solutions on PF and the position of knee regions.

nique to enhance the mmWave MIMO system.

In the following, we study the existence of a knee area for the proposed algorithm. Fig. 4 shows the relationship between the NMSE, the measurement error term and the sparse constraint term in the corresponding solutions. The corresponding left-hand column in Fig. 4 shows 3-D views of the three variables together. The right-hand column of Fig. 4 depicts 2-D plots of the measurement error $\|\boldsymbol{y}-\boldsymbol{\Phi} \boldsymbol{h}\|_{2}^{2}$ with change in sparsity. Fig. 4(b) and Fig. 4(c) show the horizontal position (sparsity axis) of knee points varies with different sparse values. In particular, under the high SNR levels, we can notice that the sparsity estimation rapidly deteriorates for points to the right of the knee region, while providing a little improvement in measurement error. It is indicated that the optimal solution can be provided in the knee region. The above results indicate that the solutions in the knee areas are consistent with the true sparsity of the original channel.

In Fig. 5, we further analyze how the system achievable spectral efficiency performance is affected by the number of IRS elements in the mmWave communication system. It is observed that the SE performance gains achieved by the IRS scheme over the 'without IRS' scheme, especially for the large number of phase shifters $M$. This is mainly due to the fact that an additional strong link is reflected by the IRS, which can be harvested by the UE. These results also demonstrate that the IRS is effective in expanding the operational range of UEs. In 


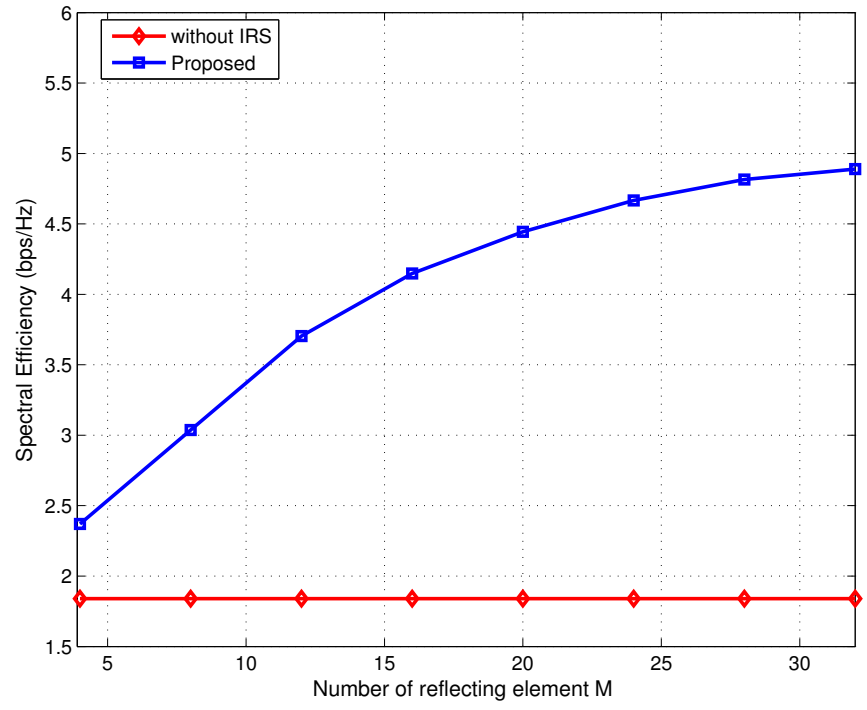

Fig. 5: Performance of IRS-aided MIMO communication versus the number of reflecting element $M$.

addition, as expected, the performance of the method "without IRS' is not affected by the number of the IRS elements in the system.

\section{CONClusion}

In this paper, we studied the mmWave channel estimation problem for IRS-assisted MIMO systems. Under the proposed framework, the cascade channel estimation approach is developed using the properties of Kronecker products. By exploiting the inherent sparse structure of the mmWave channel, we formulate the cascaded channel estimation problem into a CSbased sparse recovery problem that can achieve a substantial training overhead reduction. Under the CS-based channel estimation problem, a multiobjective evolutionary paradigm with DE is developed to achieve high resolution channel estimation. Furthermore, we provide the convergence analysis for the hybrid evolutionary-based channel estimation algorithm. Simulation results were provided to verify the effectiveness of our framework on mmWave channel.

\section{REFERENCES}

[1] S. Liu, Z. Gao, J. Zhang, M. Di Renzo, and M. Alouini, "Deep denoising neural network assisted compressive channel estimation for mmwave intelligent reflecting surfaces," IEEE Transactions on Vehicular Technology, vol. 69, no. 8, pp. 9223-9228, 2020.

[5] J. Tang, A. Shojaeifard, D. K. C. So, K. Wong, and N. Zhao, "Energy efficiency optimization for CoMP-SWIPT heterogeneous networks," IEEE Transactions on Communications, vol. 66, no. 12, pp. 6368-6383, 2018.
[2] Q. Wu and R. Zhang, "Towards smart and reconfigurable environment: Intelligent reflecting surface aided wireless network," IEEE Communications Magazine, vol. 58, no. 1, pp. 106-112, 2020.

[3] W. Yan, X. Yuan, Z. He, and X. Kuai, "Passive beamforming and information transfer design for reconfigurable intelligent surfaces aided multiuser mimo systems," IEEE Journal on Selected Areas in Communications, vol. 38, no. 8, pp. 1793-1808, 2020.

[4] J. Tang, D. K. C. So, N. Zhao, A. Shojaeifard, and K. Wong, "Energy efficiency optimization with SWIPT in MIMO broadcast channels for internet of things," IEEE Internet of Things Journal, vol. 5, no. 4, pp. 2605-2619, 2018

[6] Z. Gao, L. Dai, Z. Wang, and S. Chen, "Spatially common sparsity based adaptive channel estimation and feedback for FDD massive MIMO," IEEE Transactions on Signal Processing, vol. 63, no. 23, pp. 61696183, 2015.

[7] X. Rao and V. K. N. Lau, "Compressive sensing with prior support quality information and application to massive MIMO channel estimation with temporal correlation," IEEE Transactions on Signal Processing, vol. 63, no. 18, pp. 4914-4924, 2015.

[8] A. Taha, M. Alrabeiah, and A. Alkhateeb, "Enabling large intelligent surfaces with compressive sensing and deep learning," arXiv: Information Theory, 2019.

[9] K. Venugopal, A. Alkhateeb, N. González Prelcic, and R. W. Heath, "Channel estimation for hybrid architecture-based wideband millimeter wave systems," IEEE Journal on Selected Areas in Communications, vol. 35, no. 9, pp. 1996-2009, 2017.

[10] X. Lin, S. Wu, C. Jiang, L. Kuang, J. Yan, and L. Hanzo, "Estimation of broadband multiuser millimeter wave massive MIMO-OFDM channels by exploiting their sparse structure," IEEE Transactions on Wireless Communications, vol. 17, no. 6, pp. 3959-3973, 2018.

[11] Z. Chen, Y. Fu, Y. Xiang, and R. Rong, "A novel iterative shrinkage algorithm for CS-MRI via adaptive regularization," IEEE Signal Processing Letters, vol. 24, no. 10, pp. 1443-1447, 2017.

[12] A. Liu, V. K. N. Lau, and M. L. Honig, "Compressive RF training for massive MIMO with channel support side information," IEEE Transactions on Wireless Communications, vol. 18, no. 7, pp. 36283641, 2019.

[13] Y. Bai, Z. Qi, W. Zhihai, and Z. J. Andrew, "Adaptive decompositionbased evolutionary approach for multiobjective sparse reconstruction," Information Sciences, vol. 462, pp. 141-159, 2018.

[14] Z. Dong and W. Zhu, "Homotopy methods based on $l_{0}$-norm for compressed sensing," IEEE Transactions on Neural Networks and Learning Systems, vol. 29, no. 4, pp. 1132-1146, 2018.

[15] X. Li, S. Jin, H. A. Suraweera, J. Hou, and X. Gao, "Statistical 3-D beamforming for large-scale MIMO downlink systems over rician fading channels," IEEE Transactions on Communications, vol. 64, no. 4, pp. 1529-1543, 2016.

[16] Z. Tan, P. Yang, and A. Nehorai, "Joint sparse recovery method for compressed sensing with structured dictionary mismatches," IEEE Transactions on Signal Processing, vol. 62, no. 19, pp. 4997-5008, 2014.

[17] A. K. Qin, V. L. Huang, and P. N. Suganthan, "Differential evolution algorithm with strategy adaptation for global numerical optimization," IEEE Transactions on Evolutionary Computation, vol. 13, no. 2, pp. 398-417, 2009.

[18] C. Cartis and A. Thompson, "A new and improved quantitative recovery analysis for iterative hard thresholding algorithms in compressed sensing," IEEE Transactions on Information Theory, vol. 61, no. 4, pp. 2019-2042, 2015.

[19] L. Li, X. Yao, R. Stolkin, M. Gong, and S. He, "An evolutionary multiobjective approach to sparse reconstruction," IEEE Transactions on Evolutionary Computation, vol. 18, no. 6, pp. 827-845, 2014.

[20] X. Li, J. Fang, H. Li, and P. Wang, "Millimeter wave channel estimation via exploiting joint sparse and low-rank structures," IEEE Transactions on Wireless Communications, vol. 17, no. 2, pp. 1123-1133, 2018. 